\title{
Impact of airflow on body cooling in exercise: an exploratory study
}

\author{
Pedro H. Nogueira' , Alisson G. da Silva ${ }^{1,2}$, Samuel A. Oliveira' , Manuel Sillero-Quintana ${ }^{3}$, João C. Marins ${ }^{1}$ \\ "Universidade Federal de Viçosa. Departamento de Educação Física. Viçosa. Minas Gerais. Brasil. ${ }^{2}$ Escola Preparatória de Cadetes do Ar. Barbacena. Minas Gerais. Brasil. ${ }^{3} U$ niversidad \\ Politécnica de Madrid. Facultad de Ciencias de la Actividad Física y del Deporte (INEF). Madrid. España.
}

doi: 10.18176/archmeddeporte.00050

Recibido: $11 / 09 / 2020$ Aceptado: 20/04/2021

Key words:

Thermography. Convection. Exercise. Body temperature regulation.

\section{Summary}

Objective: To analyze the body heat dissipation by thermography during indoor running treadmill with different airflow conditions.

Materials and method: Nine male participants (23.0 2.5 years old) underwent three 45 -minute moderate-intensity running sessions (60-70\% reserve heart rate) on a treadmill. At each session, a different experimental condition was applied in a crossover design: without airflow (NF), and with low (LF) and high airflow (HF) generated by a fun. Thermograms were obtained with a thermal camera before exercise, during (every 10 minutes), and after exercise. Skin temperature (Tsk) was measured on regions of interest of the upper body: pectoral, brachial biceps, and upper back. A repeated measures ANOVA was used to compare Tsk over time and between conditions, considering $p<0.05$ as statistically significant.

Results: In pectoral and brachial biceps, LF and HF conditions provided greater reductions in Tsk at all moments when compared to the NF ( $p<0.05)$. There was a higher reduction in Tsk to the HF vs LF in biceps at 30, 40, and 45 min during exercise $(p<0.05)$. In the upper back, Tsk remained below baseline at all moments during exercise only in the HF condition $(p<0.05)$. In NF and LF conditions, Tsk returned to baseline at 30 min during exercise ( $p>0.05)$.

Conclusion: The frontal wind flow enhances body heat dissipation during moderate-intensity running in the pectoral, brachial biceps, and upper back, with a direct relationship of flow speed and Tsk reduction during exercise.

\section{Impacto del flujo de aire en el enfriamiento del cuerpo en el ejercicio: un estudio exploratorio}

\section{Resumen}

Objetivo: Analizar el disipación del calor corporal mediante termografía en ejercicio de carrera en tapiz con diferentes condiciones de flujo de aire.

Material y método: Nueve hombres (23,0 2,5 años) se sometieron a tres sesiones de 45 minutos de carrera de intensidad moderada (60-70\% frecuencia cardíaca de reserva) en tapiz, bajo tres condiciones diferentes en un diseño cruzado: sin flujo de aire (NF) y con flujo de aire bajo (LF) y alto (HF) generado por un ventilador. Los termogramas se obtuvieron con una cámara térmica antes del ejercicio, durante y después del ejercicio, midiéndose la temperatura de la piel (Tp) en las regiones de interés del pectorales, bíceps braquiales y parte superior de la espalda. Se utilizó un ANOVA de medidas repetidas para compararTp en función del tiempo y entre condiciones, considerando $p<0,05$ como estadísticamente significativo.

Resultados: En los pectorales y bíceps braquiales, las condiciones LF y HF redujeron en todo momento la Tp comparadas con NF $(p<0,05)$. La reducción de Tp fue mayor con HF que con LF en bíceps a los 30, 40 y $45 \mathrm{~min}(p<0,05)$. En la espalda superior, la Tp se mantuvo siempre por debajo de la línea base durante el ejercicio solo en la condición HF $(p<0,05)$. En condiciones de NF y LF la Tp volvió a valores de referencia a los 30 min de ejercicio ( $p>0,05)$.

Palabras clave:

Termografia. Convección. Ejercicio. Regulación de la temperatura corporal.
Conclusiones: El flujo de viento frontal mejora la disipación del calor corporal en pectorales, bíceps braquiales y parte superior de la espalda durante la carrera de intensidad moderada, con una relación directa de la velocidad del flujo y la reducción de la Tp durante el ejercicio. 


\section{Introduction}

Physical exercise is characterized by high levels of internal heat metabolic production due to muscular metabolism, which must be dissipated from the body surface to the environment to prevent dangerous elevations in internal temperature. Heat dissipation occurs through the physical processes of conduction, convection, radiation, and evaporation'. When body temperature is controlled by evaporation of sweat, caution is needed to avoid a high degree of dehydration. This condition may impair performance and health. In extreme conditions, dehydration can lead to death².

Since the airflow removes heat from the body surface, the presence of wind during exercise may assist convective heat loss ${ }^{3}$, reducing the risk of dehydration. Several studies have searched for the effects of cooling before and during exercise by using cold necklaces ${ }^{4}$, cold vests ${ }^{5}$, and head ventilators with or without water misting ${ }^{6}$. Stevens and collaborators ${ }^{7}$ reported that cooling during exercise (mid-cooling) appears largely irrelevant to core temperature reduction but may be of greater relevance in the behavior of the cardiovascular and central nervous system and some psychophysiological factors, mainly in hot environments.

Moreover, the effect of airflow at different temperatures on the face has been a quite important topic in the literature for many years. Effects of face cooling have been reported on metabolism ${ }^{8}$, heart rate $(H R)^{9}$, rate of perceived exertion (RPE) ${ }^{10}$, and energy expenditure ${ }^{11}$; however, there is a lack of studies searching on the effect of whole-body cooling by an airflow generated with a fun. Infrared thermography (IRT) is a non-invasive tool to measure the body surface radiation temperature in real time ${ }^{12}$. Although several studies have used IRT for monitoring thermoregulatory adjustments on the body surface during different types of exercise ${ }^{13-17}$, the impact of wind on the cooling capacity of the skin has not yet been studied by IRT.

The practice of indoor physical activity on a treadmill promotes a greater difficulty of convective heat loss when compared to outdoor running. Thus, the use of a fan can facilitate heat exchange through convection. Observing the skin temperature (Tsk) response during exercise with and without airflow can help to understand the importance of convection on thermoregulation. Moreover, this study can assist in the layout of spaces or in planning ventilation strategies to improve thermoregulation during exercise. Therefore, this study analyzed the body heat dissipation by thermography during indoor running treadmill with different airflow conditions.

\section{Material and method}

\section{Participants}

This study included nine healthy and physically active men, used to train on a treadmill (age $23.0 \pm 2.5$ years old, $\mathrm{VO}_{2 \max } 49.4 \pm 4.3 \mathrm{ml}(\mathrm{kg}$. min) $)^{-1}$, height $174.1 \pm 3.2 \mathrm{~cm}$, body mass $70.6 \pm 4.6 \mathrm{~kg}$, body fat $11.1 \pm$ 4.7\%). All participants presented negative responses to all questions from the PAR-Q ${ }^{18}$ and below-average coronary risk ${ }^{19}$. Several influencing factors in Tsk ${ }^{20}$ were considered as exclusion criteria: smoking, muscu- loskeletal injuries, burns on the skin; pain symptoms; fever in the last week; treatments using creams, ointments or lotions; consumption of antipyretics and/or diuretics, or any food supplement. This study was assessed by the Local Committee of Ethics in Research and conducted following ethical principles of the Declaration of Helsinki and Resolution 466/2012 of the National Health Council. Participants provided written informed consent to participate in the study.

\section{Experimental design}

In this crossover study, participants underwent three equal moderate-intensity indoor running sessions, with 2 to 7 interval days between them. In each session, a different frontal wind speed was randomly applied via fan: low flow (LF: - $1.8 \mathrm{~m} / \mathrm{s}$ ), high flow (HF: - $3.0 \mathrm{~m} / \mathrm{s}$ ), and a control condition without airflow (NF: - $0 \mathrm{~m} / \mathrm{s}$ ). To analyze the impact of airflow on body heat dissipation, the upper-body Tsk was measured from thermograms taken before, during, and after exercise.

\section{Procedures}

To mitigate circadian variations ${ }^{21}$, each participant was evaluated on the same time day. On the first day, all subjects underwent a maximal treadmill test using the Bruce protocol22 to obtain both the predicted$\mathrm{VO}_{2 \max }$ and maximum HR (Polar $\left.{ }^{\oplus}, \mathrm{F} 1+\right)$. At least two days after the test, the participants returned to the laboratory to establish the running speed of the exercise protocol for the following training HR zones: 40-50\%, $50-60 \%$, and $60-70 \%$ of reserve HR. Exercise intensity was determined according to Karvonen equation, which considers a percentage of the reserve $H R$ to calculate exercise intensity: $\left[\left(\mathrm{HR}_{\text {TRAINING }}=H R_{\text {MAXIMUM }}-H R_{\text {RES- }}\right.\right.$ TING $) \times \%$ of intensity $\left.+\left(H R_{\text {RESTING }}\right)\right]^{23}$. The maximum HR obtained in the Bruce test was used in the Karvonen equation, along with the resting HR determined after 5 minutes of lying rest (Polar ${ }^{\circledast}, \mathrm{F} 1+$ ).

On the third, fourth and fifth days of collection, the running session lasting 45 minutes was applied on a treadmill (Embreex ${ }^{\oplus}, 565$ TX-1), divided as follows: 10 minutes of warm-up (5 minutes: $40-50 \%$ of HRR $R_{\text {RESERVE }}$ at $6.6 \pm 0.6 \mathrm{~km} / \mathrm{h}$, and 5 minutes: $50-60 \%$ of HR $\mathrm{RESERVE}_{\text {E }}$ at $7.2 \pm$ $0.8 \mathrm{~km} / \mathrm{h}$ ), a main part of 30 minutes $\left(60-70 \%\right.$ of $H R_{\text {RESERVE }}$ at $8.0 \pm 1.0$ $\mathrm{km} / \mathrm{h}$ ), 5 minutes of active recovery (walking at $<50 \%$ of $H R_{\text {RESERVE }}$ ) and 5 minutes of passive recovery (standing on the treadmill). The distance covered during both the warm-up and the main part of the exercise was $571.3 \pm 62.6 \mathrm{~m}$ and $4000 \pm 519.6 \mathrm{~m}$, respectively. HR was recorded every 2 minutes, and RPE was reported every 10 minutes on the Borg 6-20 scale ${ }^{24}$. To produce the wind, a 3-propeller fan (Arno ${ }^{\oplus}$, Versatile) was positioned on the floor, one meter from the volunteer, and in front of the treadmill. The airflow was positioned from bottom to top towards the anterior upper body. The fan was turned off after completion of the exercise. Wind speed was monitored with a digital anemometer (Instrutherm, AD-250). Fan position is presented in Figure 1.

In each session, $3 \mathrm{ml}$ of water per kilogram of body mass was administered every 15 minutes of exercise as a hydration protocol ${ }^{25}$. Participants were weighed at the beginning and immediately after sessions to assess hydration status ${ }^{2}$.

The Thermographic Imaging of Sport and Exercise Medicine consensus statement was followed ${ }^{26}$. The temperature and humidity of 
Figure 1. Fan position.

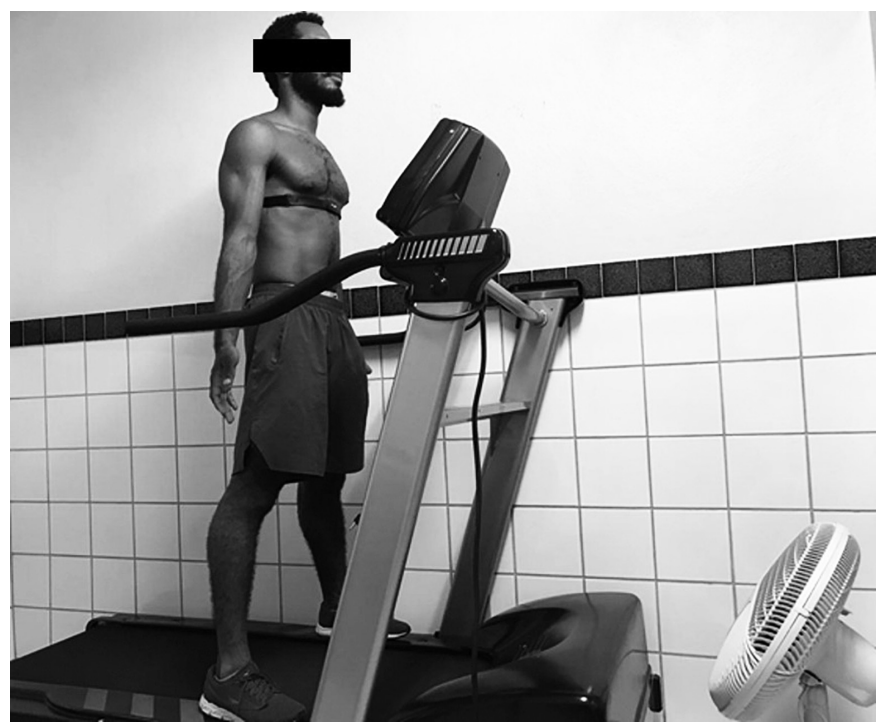

Table 1. Rate of perceived exertion during each experimental condition.

\begin{tabular}{|c|c|c|c|c|c|}
\hline \multirow{6}{*}{$\begin{array}{l}\text { Rate of } \\
\text { perceived } \\
\text { exertion } \\
(6-20)\end{array}$} & \multicolumn{5}{|c|}{ Exercise protocol } \\
\hline & \multicolumn{3}{|c|}{ Warm-up } & \multicolumn{2}{|l|}{ Main part } \\
\hline & Condition & $10 \mathrm{~min}$ & $20 \mathrm{~min}$ & $30 \mathrm{~min}$ & $40 \mathrm{~min}$ \\
\hline & Windless & $10.4 \pm 1.6$ & $12.3 \pm 1.7$ & $12.7 \pm 2.1$ & $13.3 \pm 1.5$ \\
\hline & Low flow & $10.3 \pm 1.4$ & $11.8 \pm 1.8$ & $12.5 \pm 2.0$ & $12.9 \pm 2.3$ \\
\hline & High flow & $10.8 \pm 1.2$ & $12.4 \pm 2.1$ & $13.5 \pm 2.3$ & $13.7 \pm 2.3$ \\
\hline
\end{tabular}

the room were stable between conditions [NF $\left(21.22 \pm 1.15^{\circ} \mathrm{C}, 61.33 \pm\right.$ $3.95 \%), \mathrm{LF}\left(21.17 \pm 1.32{ }^{\circ} \mathrm{C}, 62.22 \pm 4.15 \%\right), \mathrm{HF}\left(20.90 \pm 1.55^{\circ} \mathrm{C}, 60.00\right.$ $\pm 4.27 \%)$ ]. Environmental conditions were monitored using a portable weather station (Instrutherm ${ }^{\oplus}$, THAL-300), which was positioned $1.8 \mathrm{~m}$ from the floor. An acclimation of 10 minutes was performed ${ }^{27}$. At that time, subjects only wore shoes and shorts running, and the heart monitor; they were asked to remain upright on the treadmill, not to do sudden movements, and not to rub with their hands any part of the body. Thermograms (anterior and posterior upper body) were obtained before exercise; at 10, 20, 30, 40 min during exercise; and after 5 min of active recovery and $5 \mathrm{~min}$ after completion of the protocol (passive recovery). For all images, participants positioned himself in a demarcated location on the treadmill (see supplementary material).

To record thermograms, a FLIR ${ }^{\oplus}$ T420 imager was used with $2 \%$ accuracy, sensitivity $\leq 0.05^{\circ} \mathrm{C}$, at $7.5-13 \mu \mathrm{m}$ infrared spectral band, 60 $\mathrm{Hz}$ refresh rate, autofocus, and $320 \times 240$ pixels. The camera was turned on 15 min before the measures to allow for sensor stabilization. It was positioned on a tripod behind the treadmill, 2 meters away from the volunteer (see supplementary material), perpendicular to the regions of interest (ROIs) (1.5 to $1.6 \mathrm{~m}$ away from the floor ranging in accordance with the participants' height). Thermograms were analyzed using FLIR ${ }^{\circledast}$ Tools software, version 6.4, where the Tsk on ROIs of the pectoral, brachial
Figure 2. Delimitation of the upper dorsal (1), pectoralis, and biceps brachii (2) ROI.

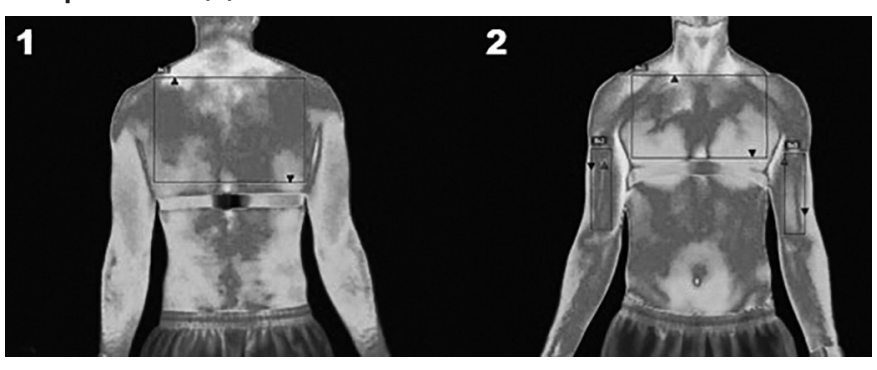

biceps, and upper dorsal were measured using anatomical points as reference: a) pectoral - the line of the nipple and the upper border of the sternum; b) biceps - the cubital fossa and the axillary line; c) upper dorsal - acromial edge of scapula and $1 \mathrm{~cm}$ above the lower scapula angle. The HR belt was out of all selected ROIs. Emissivity was set at 0.98. Figure 2 shows the demarcation of ROls.

\section{Statistical analysis}

The average Tsk obtained from each $\mathrm{ROI}$ was considered for the statistical analysis. For the arms, the arithmetic mean between the right and left side was calculated as the final value for the data analysis. The normal distribution of the data was verified by the Shapiro-Wilk test. Therefore, all data are reported as means and standard deviations. To compare the RPE among the different experimental conditions, oneway ANOVA was used. A repeated-measures ANOVA was performed to analyze Tsk changes over time and to compare the Tsk intra-moment among the different conditions. When significant $F$ values were found, Bonferroni's post hoc test was used to determine the level of significance. Sphericity was assessed by Mauchly test, and once violated, the Greenhouse-Geisser adjustment was used. The partial eta square $\left(n p^{2}\right)$ was used as a measure of effect size and classified as weak $(<0.1)$, modest (0.1-0.29), moderate (0.3-0.5), or strong (>0.5). Statistical significance was considered at $p<0.05$. All calculations were performed in SPSS, version 25.

\section{Results}

The participants' hydration status was maintained after each exercise session. Body mass changes were minimal after NF $(\Delta: 0.11 \%), \operatorname{LF}(\Delta$ : $0.05 \%)$, and $H F(\Delta: 0.16 \%)$ sessions. Regarding RPE, there was no significant difference between groups when each time point was compared (10 min, $p=0.787 ; 20$ min, $p=0.728 ; 30$ min, $p=0.563 ; 40$ min, $p=0.711$ ). Table 1 shows the rating of perceived exertion for the different protocols.

When comparing resting Tsk among the conditions, there was no significant difference: pectoral ( $p=0.158)$, upper dorsal $(p=0.137)$, or biceps brachial ( $p=0.056$ ). Next, the Tsk response of each $\mathrm{ROI}$ is presented.

\section{Pectoral}

Figure 3 shows the pectoral Tsk response to the experimental protocols. 
Figure 3. Tsk behavior $\left({ }^{\circ} \mathrm{C}\right)$ in the pectoral region.

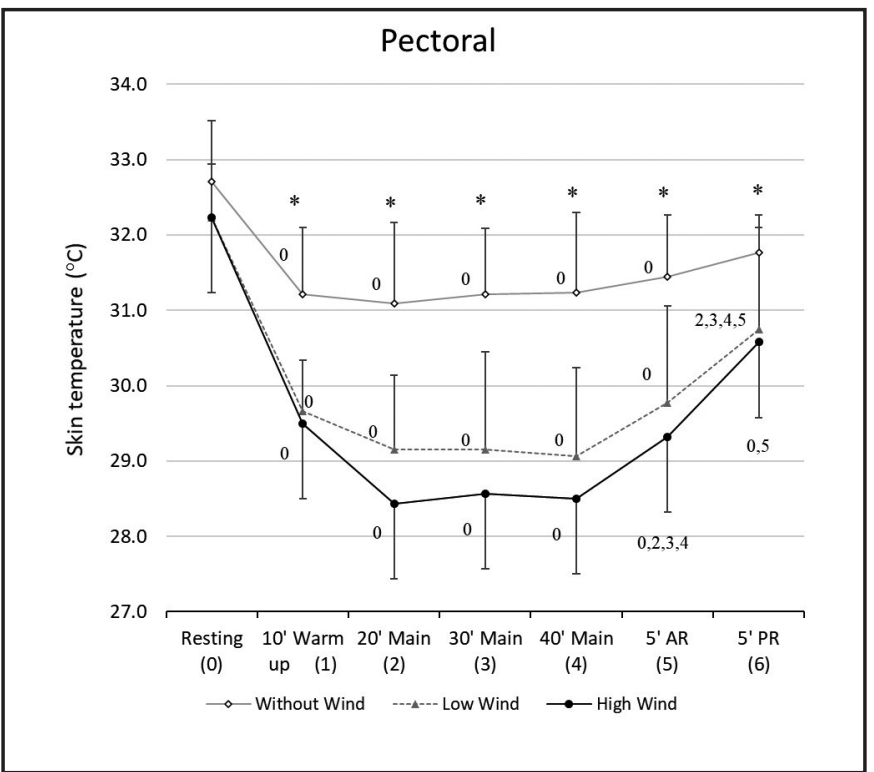

* Significant differences between groups comparing windless condition with both windy conditions. "no.": significant differences in relation to the corresponding moment condition (see code in the $\mathrm{X}$ axis).

Significant differences were found in pectoral Tsk when each moment was compared between conditions ( $p<0.001)$. In all moments, both wind speeds provided greater reductions in Tsk versus NF condition [during exercise and after active recovery ( $p \leq 0.001)$; after passive recovery (LF vs NF, $p=0.043$; HF vs NF $p=0.001$ )].

There were Tsk changes over time in the NF exercise $[F=12.389$; $\left.p<0.001, \eta p^{2}=0.608\right]$. Tsk decreased after 10 min of exercise $(p=0.001)$ and was sustained below baseline throughout the session $(p \leq 0.01)$ and after $5 \mathrm{~min}$ of active recovery $(p=0.015)$. After $5 \mathrm{~min}$ of passive recovery, Tsk returned to baseline ( $p=0.078)$.

In the LF condition, Tsk changed over time $[F=26.708 ; p<0.001$, $\left.\eta p^{2}=0.770\right]$. Tsk decreased after 10 min of exercise $(p<0.001)$ and was kept below baseline throughout the session $(p \leq 0.01)$ and after 5 min of active recovery ( $p=0.015)$. However, after $5 \mathrm{~min}$ of passive recovery, Tsk returned to baseline ( $p=0.188$ ). Moreover, Tsk increased after $5 \mathrm{~min}$ of passive recovery when compared to 20,30, $40 \mathrm{~min}$, and after active recovery $(p \leq 0.01)$.

In the HF session, there were significant Tsk changes over time $\left[F=47.980 ; p<0.001, n p^{2}=0.857\right]$. Tsk decreased after 10 min of exercise $(p<0.001)$ and was kept below baseline throughout the session $(p<0.001)$, after 5 min of active recovery $(p=0.001)$ and after 5 min of passive recovery $(p=0.007)$. However, after $5 \mathrm{~min}$ of active recovery, Tsk increased when compared to after 20,30, and 40 min of exercise $(p<0.05)$. Furthermore, Tsk increased after 5 min of passive recovery compared to 20,30, 40 min of exercise, and 5 min of active recovery $(p<0.001)$.

\section{Brachial biceps}

Figure 4 shows the biceps Tsk response to the experimental protocols.
Figure 4. Tsk behavior $\left({ }^{\circ} \mathrm{C}\right)$ in the biceps region.

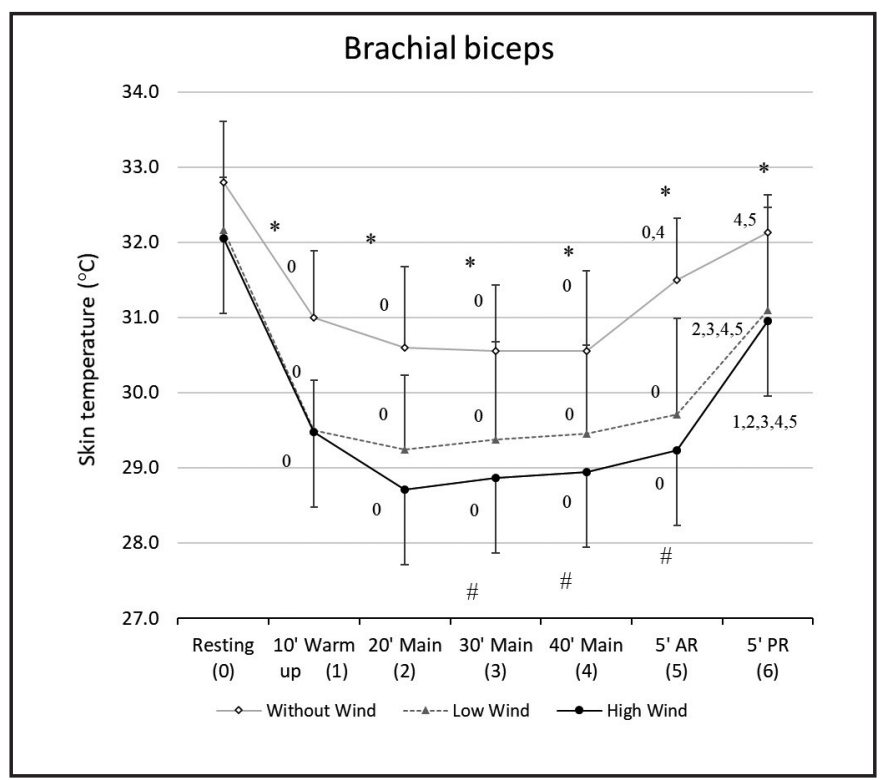

* Significant difference between groups in the comparison between the without wind condition (NF) vs little airflow (LF) and high flow (HF). \# significant difference between groups when comparing low wind vs high wind conditions." no.": significant differences in relation to the corresponding moment condition (see code in the X axis).

Significant differences were found in biceps Tsk when each moment was compared between conditions ( $p \leq 0.001$ ). In all moments, both wind speeds provided greater reductions in Tsk versus NF condition $(p \leq 0.01)$. Moreover, the HF versus LF condition provided a greater reduction in Tsk after 30,40 , and 5 min of active recovery $(p<0.05)$.

There were Tsk changes over time in the NF exercise $[F=54.555$; $\left.p<0.001, \eta p^{2}=0.872\right]$. Tsk decreased after 10 min of exercise $(p<0.001)$ and was sustained below baseline throughout the session $(p \leq 0.001)$. However, after 5 min of passive recovery, Tsk returned to rest ( $p=0.186$ ). Moreover, Tsk increased after 5 min of active recovery ( $p=0.001)$ and after passive recovery $(p<0.001)$ when compared to the time of completion of the main part of the exercise (40 min). Finally, Tsk increased after 5 min of passive recovery versus after active recovery $(p=0.001)$.

In the LF session, Tsk changed over time [F=27.943; $p<0.001$, $\left.\eta p^{2}=0.777\right]$. Tsk decreased after 10 min $(p<0.001)$ of exercise and was kept below baseline throughout the main part of the session $(p \leq 0.01)$ and after active recovery $(p=0.034)$. However, after 5 min of passive recovery, Tsk returned to rest ( $p=1.0)$. Furthermore, Tsk increased after passive recovery when compared to after 20 ( $p=0.022), 30,40 \mathrm{~min}$, and active recovery $(p<0.001)$.

In the HF session, there were significant Tsk changes across time $\left[\mathrm{F}=54.484 ; p<0.001, n p^{2}=0.872\right]$. Tsk decreased with 10 min of exercise ( $p=0.001)$ and was kept below baseline throughout the session $(p<0.001)$ and after passive recovery $(p=0.018)$. However, after passive recovery, Tsk increased when compared to the 10 ( $p=0.018), 20(p=0.027)$, and other moments throughout the exercise $(p<0.001)$.

\section{Upper dorsal}

Figure 5 shows the dorsal Tsk response to the experimental protocols. 
Figure 5. Skin temperature behavior $\left({ }^{\circ} \mathrm{C}\right)$ in the dorsal region.

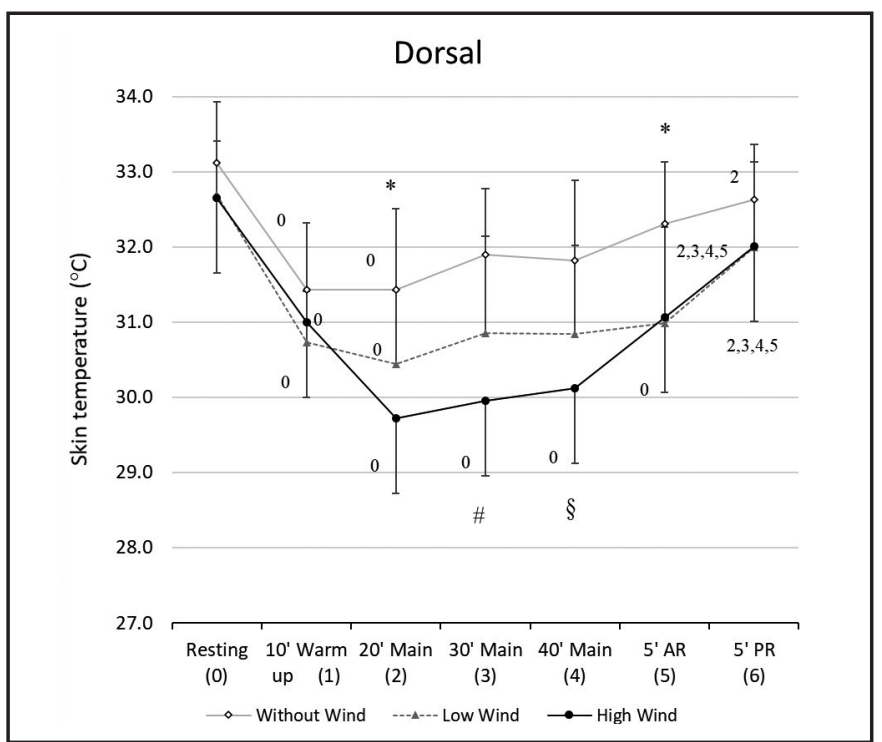

* Significant difference between groups in the comparison between the condition without wind vs little wind and a lot of wind. \# Significant difference between groups when comparing the very wind vs no wind and little wind conditions. § Significant difference between groups when comparing windless vs very windy conditions. "no": significant differences in relation to the corresponding moment condition (see code in the $\mathrm{X}$ axis).

Significant differences were found in dorsal Tsk when the time points (20, 30, 40 min, and active recovery) were compared between conditions ( $p \leq 0.001)$. Both wind speeds provided greater reductions in Tsk when compared to the NF condition at $20 \mathrm{~min}$ and after active recovery $(p \leq 0.01)$. Moreover, the HF condition provided a greater reduction in Tsk at 30 min versus LF $(p=0.043)$ and NF $(p<0.001)$ conditions and at 40 min versus NF condition $(p<0.001)$.

There were Tsk changes over time in the NF exercise $[\mathrm{F}=8.787$; $\left.p<0.001, n p^{2}=0.523\right]$. Tsk decreased after $10(p=0.003)$ and 20 min
( $p=0.038$ ) of exercise compared to baseline. However, Tsk returned to baseline after 30 min of exercise $(p=0.081)$ and stabilized until the end of exercise $(p>0.05)$. Furthermore, Tsk after passive recovery was higher than 20 min of exercise $(p=0.049)$.

In the LF session, Tsk changed over time $[F=8.271 ; p=0.007$, $\left.\eta p^{2}=0.508\right]$. Tsk decreased after $10(p=0.004)$ and $20 \mathrm{~min}(p=0.023)$ of exercise versus baseline. However, Tsk returned to baseline after 30 min of exercise ( $p=0.325)$. Moreover, Tsk after passive recovery was higher than after 20, 30, 40 min, and 5 min of active recovery $(p<0.05)$.

Finally, in exercise session $\mathrm{HF}$, Tsk changed over time $[\mathrm{F}=40.406$; $\left.p<0.001, n p^{2}=0.835\right]$. Tsk decreased after 10 min of exercise $(p=0.008)$ and was kept below baseline throughout the running session $(p \leq 0.001)$ and after active recovery $(p=0.004)$. However, after passive recovery, Tsk returned to baseline ( $p=0.706)$. Additionally, Tsk after passive recovery was higher compared to all moments during and after exercise $(p \leq 0.001)$.

\section{Exercise-induced Tsk reduction with and without wind}

Table 2 shows the average Tsk reduction in each experimental condition.

\section{Distribution of Tsk under experimental conditions}

From a qualitative perspective, Figure 6 shows the Tsk distribution on the body surface for one participant before exercise, after 40 min of exercise, and after 5 minutes of passive recovery, in each experimental condition. It is possible to highlight the similarity between the preexercise thermograms, as well as the clear Tsk reduction, especially in the anterior region of the trunk and arms after 40 min of exercise mainly in conditions with the presence of wind. In the recovery period of the NF exercise, the thermogram became very similar to the resting thermogram, while thermal reduction remained evident under windy conditions. Characteristic hot spots are observed in the central region of the chest, back, and arms after 40 min and in recovery from exercise; this phenomenon was attenuated according to the intensity of the wind.

Table 2. Average thermal reduction $\left(\Delta \mathrm{T}^{\circ} \mathrm{C}\right)$ at each moment compared to baseline Tsk.

\begin{tabular}{|c|c|c|c|c|c|c|c|c|}
\hline \multirow[b]{3}{*}{ ROI } & \multirow[b]{3}{*}{ Condition } & \multirow[b]{3}{*}{$\begin{array}{c}\text { Basal } \\
\text { Tsk }\left({ }^{\circ} \mathrm{C}\right)\end{array}$} & \multicolumn{6}{|c|}{ Exercise protocol } \\
\hline & & & \multirow{2}{*}{$\begin{array}{c}\text { Warm-up } \\
10 \text { min } \\
\Delta T^{\circ} \mathrm{C}\end{array}$} & \multicolumn{3}{|c|}{ Main part } & \multicolumn{2}{|c|}{ Recovery } \\
\hline & & & & $\begin{array}{c}20 \min \\
\Delta T^{\circ} \mathrm{C}\end{array}$ & $\begin{array}{l}30 \min \\
\Delta T^{\circ} \mathrm{C}\end{array}$ & $\begin{array}{c}40 \min \\
\Delta T^{\circ} \mathrm{C}\end{array}$ & $\begin{array}{c}5 \min A R \\
\Delta T^{\circ} \mathrm{C}\end{array}$ & $\begin{array}{c}5 \min P R \\
\Delta T^{\circ} \mathrm{C}\end{array}$ \\
\hline \multirow[t]{3}{*}{ Pectoral } & NF & 32.7 & -1.5 & -1.6 & -1.5 & -1.5 & -1.3 & -0.9 \\
\hline & LF & 32.2 & -2.6 & -3.1 & -3.1 & -3.2 & -2.5 & -1.5 \\
\hline & $\mathrm{HF}$ & 32.2 & -2.7 & -3.8 & -3.7 & -3.7 & -2.9 & -1.6 \\
\hline \multirow[t]{3}{*}{ Biceps } & NF & 32.8 & -1.8 & -2.2 & -2.2 & -2.2 & -1.3 & -0.7 \\
\hline & LF & 32.2 & -2.7 & -2.9 & -2.8 & -2.7 & -2.5 & -1.1 \\
\hline & $\mathrm{HF}$ & 32.0 & -2.5 & -3.3 & -3.2 & -3.1 & -2.8 & -1.1 \\
\hline \multirow[t]{3}{*}{ Dorsal } & NF & 33.1 & -1.7 & -1.7 & -1.2 & -1.3 & -0.8 & -0.5 \\
\hline & LF & 32.7 & -2.0 & -2.3 & -1.8 & -1.9 & -1.7 & -0.7 \\
\hline & $\mathrm{HF}$ & 32.7 & -1.7 & -2.9 & -2.7 & -2.5 & -1.6 & -0.6 \\
\hline
\end{tabular}

ROI: body region of interest; NF: windless; LF: low flow; HF: high flow; Tsk: skin temperature; AR: active recovery; PR: passive recovery; $\triangle T$ : thermal reduction compared to baseline. 
Figure 6. Evolution of the anterior and posterior thermograms of a participant in the pre-exercise period, after 40 min of exercise, and after 5 min of passive recovery moments under the three experimental conditions.

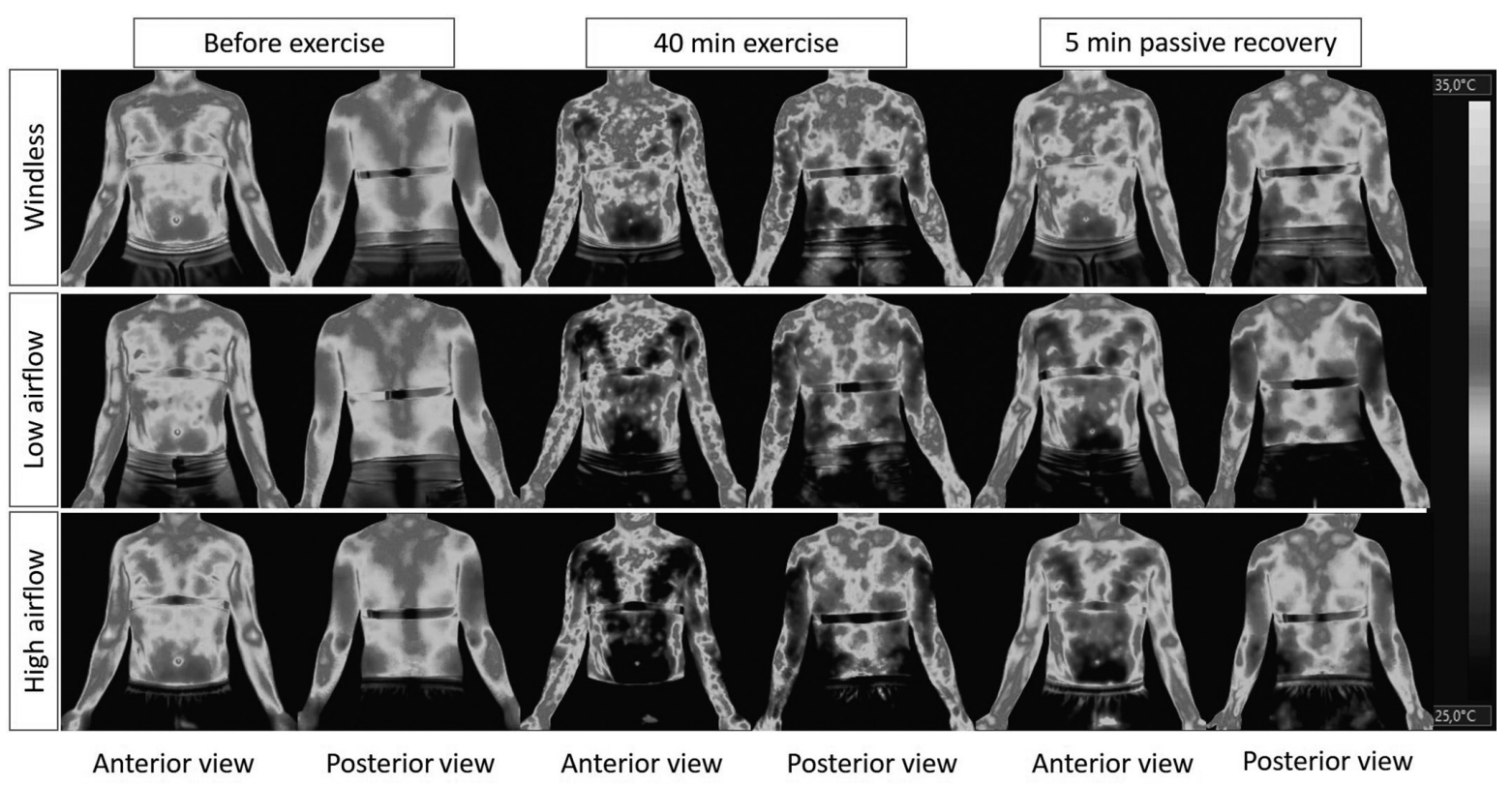

\section{Discussion}

The objective of this study was to analyze the body heat dissipation during indoor running treadmill with different airflow conditions. The pre-exercise conditions among testing days were similar regarding hydration level, environmental condition, body mass, and Tsk. Moreover, the participants' RPE during exercise was statistically similar among the different protocols. Taken together, these results reinforce that the observed thermal changes were due to the different airflow conditions during exercise. Our main findings show an important impact of wind on the body surface cooling during moderate-intensity exercise on a treadmill. This effect has been visualized and quantified with thermography.

Since we analyzed inactive muscle groups during running, the exercise-induced Tsk reduction may be attributed to adrenergic cutaneous vasoconstriction, which contributes to redirecting blood flow from inactive regions to active muscle ${ }^{28-31}$. This adjustment may explain the Tsk reduction especially in the initial moments of windless exercise $^{12}$. Other authors have reported Tsk reductions in inactive ROls during exercise $\mathrm{e}^{15,17}$. Moreover, Tsk reduction may be explained by evaporative heat loss. As exercise continues, the excess of metabolic heat needs to be transferred to the body surface, where it is dissipated to the environment through the evaporation of sweat ${ }^{29,32}$

When the use of the ventilator was included in the exercise session, the Tsk reduction was enhanced in all the ROls. This behavior accelerates the body's surface cooling, facilitating thermoregulation. In the present study, it was evident how the effect of the airflow acts in removing heat from the body through convection, cooling the sweat, or even helping in its evaporation ${ }^{3}$. This Tsk reduction favors heat dissipation to the environment, as it increases the thermal gradient between the core and the skin, facilitating internal heat transfer to the skin'. Thus, our results demonstrate the efficiency of the airflow during exercise to increase heat dissipation in running exercise. The impact of the wind in the Tsk distribution on the body surface of a participant can be seen in Figure 6 .

In the chest, the different wind speeds comparably potentiated the Tsk reduction at all moments in comparison to the condition NF. Therefore, in pectorals, a low wind speed was sufficient to maximize the release of body heat. However, the higher wind speed contributed to the maintenance of the thermal reduction after the end of the exercise, since Tsk did not return to the rest value after passive recovery only in HF condition (Figure 3).

The greatest Tsk drops in comparison with the NF condition were found in the chest. Reductions of $\approx 1.5^{\circ} \mathrm{C}$ during the NF exercise were maximized by airflow, reaching values greater than $3^{\circ} \mathrm{C}$ with $L F$ and $\approx$ $4^{\circ} \mathrm{C}$ with HF (Table 2). A probable explanation is that the chest received a greater flow of wind due to its large contact surface in comparison to the biceps; compared to the dorsal ROI, the chest (but not the dorsal) received direct wind during exercise.

In the biceps, the wind flow potentiated the reduction in Tsk throughout the exercise session in comparison to the NF condition. The greatest thermal reduction during the NF exercise was $2.2^{\circ} \mathrm{C}$, while in the LF and HF conditions, the thermal reduction reached values of $2.9^{\circ} \mathrm{C}$ and $3.3^{\circ} \mathrm{C}$, respectively. Moreover, the higher wind speed induced Tsk reductions of greater magnitude compared to the LF condition at 30 
and $40 \mathrm{~min}$ of exercise, and after 5 min of active recovery $\left(\triangle \mathrm{Tsk} \approx 0.4^{\circ} \mathrm{C}\right.$ between HF and LF) (Table 2). As with the chest, the increased participation of the convection mechanism promoted by the higher wind speed could have been important to keep the Tsk reduced longer time in the biceps after exercise.

The dorsal region did not receive direct wind. Even so, the exercise conditions with wind were effective in reducing Tsk (Figure 5) but in a less accentuated way than in the other ROIs. While the Tsk reduction in the NF exercise was 1.2 to $1.7^{\circ} \mathrm{C}$ in the main part of the exercise, under wind conditions, this reduction varied from $1.8-2.3^{\circ} \mathrm{C}$ with $L F$ and 1.7 $-2.9^{\circ} \mathrm{C}$ with HF (Table 2). These results confirm that even though the dorsal region had not received frontal airflow, the back is an important heat exchange region for the human body since it has more eccrine sweat glands compared to other regions ${ }^{33,34}$. The greater potential for sweat production in the dorsal $\mathrm{ROI}$ can increase the convective heat loss when there is wind flow in the environment.

The dorsal ROI showed a non-linear behaviour of Tsk after the end of the warm-up. However, the return of Tsk to baseline was after 30 min of exercise in the NF and LF conditions, while the thermal reduction in the $\mathrm{HF}$ condition was preserved throughout the training session, returning to baseline only after $5 \mathrm{~min}$ of passive recovery. These findings indicate that even with indirect airflow, the speed of the wind influences the cooling of this region, increasing its potential for heat loss.

In the post-exercise recovery period, there was a gradual increase in Tsk even in the presence of airflow. This response probably occurred due to the lower intensity after the end of the main part of the exercise. It has been shown that the reduction in Tsk during exercise is proportional to the increase in intensity ${ }^{16}$. Therefore, from the moment that the intensity was reduced in the recovery period, the thermal reduction previously achieved was attenuated. Moreover, after active recovery, the fun was off, which increased the Tsk after 5 minutes of passive rest. These results reinforce the relevance of wind flow on skin cooling during exercise.

Currently, many gyms have installed aerobic rooms, where several fans can be arranged to allow airflow to reach the maximum of the skin areas of the athlete. On the other hand, some treadmill models already have a fan attached, helping thermoregulation during exercise. Considering the duration of exercise (45 min) in our study, it was observed that the wind plays a fundamental role in body thermoregulation by increasing the participation of the convection mechanism, making it evident that simple fans are sufficient to enhance the body surface cooling during the exercise period.

A limitation of the study is the absence of other techniques to assess hydration status after exercise. However, since changes in the participants' body weight were lower than $2 \%$, we assume that the hydration protocol ensured a euhydration condition and avoided interference in thermoregulatory responses. The pioneering nature of our study was to quantify, by IRT, the impact of the wind on Tsk, something that has not yet been accomplished. We demonstrate the magnitude of body surface cooling during exercise with and without any airflow, confirming that convection increases the potential for heat exchange ${ }^{3}$. To replicate our study with highly trained runners is interesting since the effect of systematic training on the thermoregulation of experienced runners could provide different results. Future studies should investigate whether airflow-induced body cooling could translate into a better exercise performance, including female participants since gender influences thermoregulatory processes ${ }^{35}$.

\section{Conclusion}

The airflow during moderate-intensity treadmill running enhance the heat dissipation from the upper body surface to the environment in physically active men. In the chest, reduced wind speed is sufficient to increase the release of heat. In the brachial biceps, the higher wind speed induces thermal reductions of greater magnitude. In the upper dorsal region, the higher wind speed is effective in increasing the release of heat and keeping Tsk reduced during exercise.

\section{Acknowledgments}

Conselho Nacional de Pesquisa (CNPq); Fundação de Amparo à Pesquisa do Estado de Minas Gerais (FAPEMIG).

\section{Support received}

Conselho Nacional de Pesquisa (CNPq).

\section{Conflict of interest}

The authors do not declare a conflict of interest.

\section{Bibliography}

1. Charkoudian N. Mechanisms and modifiers of reflex induced cutaneous vasodilation and vasoconstriction in humans. J Appl Physiol. 2010;109:1221-8.

2. McDermott BP, Anderson SA, Armstrong LE, Casa DJ, Cheuvront SN, Cooper L, et al. National athletic trainers' association position statement: fluid replacement for the physically active. J Athl Train. 2017;52:877-95.

3. Parsons K. Human thermal environments: the effects of hot, moderate, and cold environments on human health, comfort, and performance. Boca Raton. CRC Press; 2014. p. 94.

4. Tyler CJ, Wild P, Sunderland C. Practical neck cooling and time-trial running performance in a hot environment. Eur J Appl Physiol. 2010;110:1063-74.

5. Racinais S, Ihsan M, Taylor L, Cardinale M, Adami PE, Alonso JM, et al. Hydration and cooling in elite athletes: relationship with performance, body mass loss and body temperatures during the Doha 2019 IAAF World Athletics Championships. Br J Sports Med. 2021;0:1-8. doi:10.1136/bjsports-2020-103613.

6. Tyler CJ, Sunderland C, Cheung SS. The effect of cooling prior to and during exercise on exercise performance and capacity in the heat: a meta-analysis. Br J Sports Med. 2015;49:7-13.

7. Stevens CJ, Taylor L, Dascombe BJ. Cooling during exercise: and overlooked strategy for enhancing endurance performance in the heat. Sports Med. 2017;47:829-41.

8. Riggs JC, Johnson DJ, Kilgour RD, Konopka BJ. Metabolic effects of facial cooling in exercise. Aviat Space Environ Med. 1983;54:22-6.

9. Riggs CE, Johnson DJ, Konopka BJ, Kilgour RD. Exercise heart rate response to facial cooling. Eur J Appl Physiol Occup Physiol. 1981;47:323-30.

10. Armada-da-Silva PA, Woods J, Jones DA. The effect of passive heating and face cooling on perceived exertion during exercise in the heat. Eur J Appl Physiol. 2004;91:563-71.

11. Stroud MA. Effects on energy expenditure of facial cooling during exercise. Eur J App/ Physiol Occup Physiol. 1991;63:376-80.

12. Hillen B, Pfirrmann D, Nägele M, Simon P. Infrared thermography in exercise physiology: the dawning of exercise radiomics. Sports Med. 2020;50:263-82.

13. Oliveira SAF, Marins JCB, Silva AG, Brito CJ, Moreira DG, Sillero-Quintana M. Measuring of skin temperature via infrared thermography after an upper body progressive aerobic exercise. JPES. 2018;18:184-92. 
14. Fernandes AA, Amorim PRS, Brito CJ, Costa CMA, Moreira DG, Sillero-Quintana M, et al. Skin temperature behavior after a progressive exercise measured by infrared thermography. JPES. 2018;3:1592-600.

15. Fernandes AA, Amorim PRS, Brito CJ, Sillero-Quintana M, Marins JCB. Regional skin temperature response to moderate aerobic exercise measured by infrared thermography. Asian J Sports Med. 2016;7:e29243.

16. Merla A, Mattei PA, Di Donato L, Romani GL. Thermal imaging of cutaneous temperature modifications in runners during graded exercise. Ann Biomed Eng. 2010;38:158-63.

17. Zontak A, Sideman S, Verbitsky O, Beyar R. Dynamic thermography: analysis of hand temperature during exercise. Ann Biomed Eng. 1998;26:998-3.

18. Shepard RJ. PAR-Q, Canadian home fitness test and exercise screening alternatives. Sports Med. 1998;5:185-95.

19. American Heart Association AHA.. Risko. Lancet. 1973;2:243-4

20. Fernández-Cuevas I, Marins JCB, Lastras JA, Carmona PMG, Cano SP, García-Concepción MA, et al. Classification of factors influencing the use of infrared thermography in humans: a review. Infrared Phys Technol. 2015;71:28-55.

21. Costa CMA, Moreira DG, Sillero-Quintana M, Brito CJ, Pussieldi GA, Fernandes AA, et al Daily rhythm of skin temperature of women evaluated by infrared thermal imaging. $J$ Thermal Biol. 2018;72:1-9.

22. Bruce RA, Blackmon JR, Jones JW, Strait G. Exercising testing in adult normal subjects and cardiac patients. Pediatrics. 1963;32:742-56.

23. Karvonen MJ, Kentala E, Mustala O. The effects of training on heart rate; a longitudinal study. Ann Med Exp Biol Fenn. 1957;35:307-15

24. Borg GA. Psychophysical bases of perceived exertion. Med Sci Sports Exerc. 1982;14:377-81.

25. Marins JCB. Hidratação na atividade física e no esporte: equilíbrio hidromineral. Várzea Paulista. Fontoura; 2011. p. 172
26. Moreira DG, Costello JT, Brito CJ, Adamczyk JG, Ammer K, Bach AJ, et al. Thermographic imaging in sports and exercise medicine: a Delphi study and consensus statement on the measurement of human skin temperature. J Thermal Biol. 2017;69:155-62.

27. Marins JCB, Moreira DG, Cano SP, Sillero-Quintana M, Soares DD, Fernandes AA, et al. Time required to stabilize thermographic images at rest. Infrared Phys Technol. 2014;65:30-5.

28. Kenny GP, JourneayWS. Human thermoregulation: separating thermal and nonthermal effects on heat loss. Front Biosci. 2010;15:259-90.

29. Smith CJ, Johnson JM. Responses to hyperthermia. Optimizing heat dissipation by convection and evaporation: Neural control of skin blood flow and sweating in humans. Auton Neurosci. 2016:196:25-36

30. Abate M, Di Carlo L, Di Donato L, Romani GL, Merla A. Comparison of cutaneous termic response to a standardised warm up in trained and untrained individuals. J Sports Med Phys Fitness. 2013;53:209-15.

31. Kenny GP, McGinn R. Restoration of thermoregulation after exercise. J Ap/l Physiol. 2017;122:933-44

32. Silva AG, Albuquerque MR, Brito CJ, Oliveira SAF, Stroppa GM, Sillero-Quintana M, et al. Resposta térmica da pele ao exercício em remoergômetro de alta versus moderada intensidade em homens fisicamente ativos. Rev Port Ciênc Desporto. 2017;17:125-37.

33. Coull NA, West AM, Hodder SG, Wheeeler P, Havenith G. Body map of regional sweat distribution in young and older males. Eur J Appl Physiol. 2021;121:109-25.

34. Taylor NA, Machado-Moreira CA. Regional variations in transepidermal water loss, eccrine sweat gland density, sweat secretion rates and electrolyte composition in resting and exercising humans. Extrem Physiol Med. 2013;2:1-29.

35. Yanovich R, Ketko I, Charkoudian N. Sex differences in human thermoregulation Physiology. 2020;35:177-84 